\title{
Severe Fever with Thrombocytopenia Syndrome
}

\author{
Seung Jin Yoo, M.D., Sang Taek Heo, M.D., and Keun Hwa Lee, M.D., Ph.D. \\ Division of Infectious Disease, Departments of Internal Medicine, and *Department of Microbiology and Immunology, \\ Jeju National University School of Medicine, Jeju, Korea
}

Severe fever with thrombocytopenia syndrome (SFTS) is a newly emerging infectious disease, caused by a novel species of Phlebovirus of Bunyaviridae family, in China, South Korea, and Japan. SFTS is primarily known as a tick-borne disease, and human-to-human transmission is also possible in contact with infectious blood. Common clinical manifestations include fever, thrombocytopenia, and leukopenia as initial symptoms, and multiple organ dysfunction and failure manifest with disease progression. Whereas disease mortality is reported to be $12 \%$ to $30 \%$ in China, a recent report of cumulative SFTS cases indicated $47 \%$ in Korea. Risk factors associated with SFTS were age, presence of neurologic disturbance, serum enzyme levels, and elevated concentrations of certain cytokines. Diagnosis of SFTS is based on viral isolation, viral identification by polymerase chain reaction, and serologic identification of specific immunoglobulin G. Therapeutic guideline has not been formulated, but conservative management is the mainstream of treatment to prevent disease progression and fatal complications.

Key Words: phlebovirus; severe fever with thrombocytopenia syndrome; Korea

\section{INTRODUCTION}

Since its first identification in China in 2006, the incidence of newly emerging infectious disease, Severe Fever with Thrombocytopenia Syndrome (SFTS), has been consistently increasing in northeastern Asian countries including China, South Korea, and Japan.[1-3] Vector of SFTS is primarily reported to be Haemaphysalis longicornis, which is also known for other tick-borne diseases such as Lyme disease, rickettsia, and spotted fever. Despite a lack of thorough understanding of disease progression, clinical manifestations initially include fever, gastrointestinal symptoms, thrombocytopenia, leukopenia, and bleeding tendency. In critically ill patients, the conditions rapidly progress to multiple organ failure and death, whose mortality re-

Received on April 24, 2014 Revised on May 7, 2014

Accepted on May 7, 2014

Correspondence to: Sang Taek Heo, Division of Infectious Disease, Department of Internal Medicine, Jeju National University School of Medicine, 15 Aran 13-gil, Jeju 690-767, Korea

Tel: +82-64-717-1296, Fax: +82-64-717-1131

E-mail: neosangtaek@naver.com ported $12 \%$ to $30 \%$.[4]

The current review is based on the collection of data reported in previous studies regarding SFTS and its virus. This paper systematically reviews epidemiology, transmission, clinical symptoms, diagnosis, and treatment. Information described herein is believed to improve understanding of the newly emerging infectious disease and virus and to help to not only prevent but also control disease progression in endemic areas.

\section{EPIDEMIOLOGY}

SFTS virus is a RNA virus, a novel Phlebovirus species in Bunyaviridae family, first retrospectively isolated in China from a 42-year-old male occurred in 2006.[5] Until 2012, the accumulated death from SFTS was described to be 129 from 2,047 infected cases in China.[6] The first reported Japanese SFTS case occurred in Yamaguichi prefecture in 2012, and a retrospective study further identified 6 more SFTS cases in western provinces of Japan.[3] The first SFTS occurrence outside Asia was identified in the United Arab Emirate from a temporary North Korean resident in 2011, which suggests a potential SFTS

cc This is an Open Access article distributed under the terms of the Creative Commons Attribution Non-Commercial License (http://creativecommons.org/ licenses/by-nc/3.0/) which permits unrestricted non-commercial use, distribution, and reproduction in any medium, provided the original work is properly cited.

(c) 2014 Korean Society of Critical Care Medicine 
infection in North Korea.[7] In South Korea, the first clinically suspected patient was detected on Jeju Island on May 2013, and the first viral isolation was reported by Seoul National University Hospital from a retrospective study.[8,9] Until November 2013, a report from Korean Center for Disease Control indicated that cumulative confirmed cases of SFTS were 36 out of 404 suspicious cases, and fatal cases were 17 in South Korea.[10]

Among the reported cases, more than $90 \%$ of patients were older than 35 years of age with a median age of 58 years, and farmers were the most affected population.[6] SFTS infection also showed seasonal distribution with biphasic peaks of incidence in May to June and August to September. Such seasonal occurrence especially in early summer and late fall seems to be attributed to increased outdoor activity.[2]

\section{ROUTE OF TRANSMISSION}

SFTS is primarily known to be a tick-borne disease, vector of which is predominantly $H$. longicornis with greater prevalence and in some cases Rhipicephalu microplus with lesser prevalence in epidemic and non-epidemic area, respectively.[6,11] A history of tick exposure was found in $52 \%$ of SFTS patients, and nucleic acid sequences of SFTS virus isolated from the ticks that were collected from mammals and environment in endemic areas showed $95 \%$ to $100 \%$ homology with that isolated from the patients.[2] Analysis of seroprevalence in mammals such as goats, cattle, and dogs infested with ticks indicated antibody positivity up to $57 \%$.[12]

Tang et al.[13] reported that human-to-human transmission in cases of unprotected contact with SFTS patients or contact with infectious blood and bodily fluid. Viral RNA was detected in blood, throat, urine, and fecal specimen.[4] Most secondary SFTS infections occurred in a family cluster, and non-familial secondary infection was reported in patients in proximity to the infected patient and healthcare workers.[14] Interpersonal transmission of SFTS virus is more probable from critically ill patients whose blood concentration of SFTS virus is increased. Reported vulnerable population was a doctor performing tracheal intubation, a consultant doctor exposed to infectious blood from intravenous lines, and a local mortuary beautician managing the corpse.[15] Secondary infection should be closely monitored at least for the incubation period ( $>13$ days) because possibility of transmission is increased more than 3 times in contact with infectious blood and in exposure of blood to mucosal membrane; therefore, personal protective measures are critical in dealing with infected patients and dead patients.[13]

\section{CLINICAL MANIFESTATION AND LABORATORY FINDINGS}

Upon time of viral transmission, incubation period of SFTS virus is reported to be 6 to 14 days, and eschar-like cutaneous lesion, usually round, crust bearing, and ulcerative, is commonly associated with tick bite.[16] Even though history of tick bite is common in SFTS infection, tick bite is not evident in some patients. Because most SFTS patients are older population, it is common that they often do not recall history of tick bite. Ticks are attracted to mammals by exhaled carbon dioxide, and because ticks spit back chemicals to prevent itching symptoms in patients after sucking host's blood, patients may not potentially sense the bites. Therefore, although history of tick bite is helpful in diagnosis of SFTS, history of outside activity should be considered more carefully.

Even though some are self-limited without any symptoms, most infected patients commonly complain of fever, myalgia, arthralgia and gastrointestinal symptoms such as nausea, vomiting, abdominal pain, and diarrhea.[5] Gai et al.[1] categorized clinical progression of SFTS into 3 stages: febrile, multiple organ dysfunction, and convalescent stages. Febrile stage is characterized by initial high serum viral load of $10^{5-6}$ copies $/ \mathrm{mL}$ and marked thrombocytopenia and leukopenia; in addition, activated partial thromboplastin time and liver enzymes begin to increase. In the febrile stage, incidences of proteinuria and hematuria are common and higher in fatal cases. In multiple organ dysfunction stage, characterized by progressive exacerbation of the disease, increased tissue enzymes drops after reaching its peaks in non-fatal cases, but they continue to rise and reach its maximal values before death in fatal cases. In convalescent stage, most of critical clinical parameters such as body temperature, hematologic values, and serum enzymes are normalized.[1] Immunologic analysis of SFTS patients revealed that elevation of CD 69+ T cells was evident in acute phase after onset of illness and that HLA-DR+ and CTLA4+ T cells were increased in the convalescent phase.[17] In addition, Deng et al.[18] reported that concentrations of TNF- $\beta$, IL 6 , IFN- $\gamma$, and IFN- $\gamma$-induced protein 10 were associated with disease severity. Takahashi et al.[3] performed bone marrow examination in fatal SFTS patients and revealed macrophages with phagocytosis in bone marrow cells; in addition, further pathologic examinations indicated marked hemophagocytosis in lymph nodes, bone marrow, and spleen.

Mortality rate is reported from $6 \%$ to $30 \%$ in previous Chinese reports, but the most up-to-dated report from Korean 
Center for Disease Control indicated mortality rate of $47.3 \%$ in cumulative 36 fatal SFTS cases occurred in 2013.[2,10] Risk factors associated with fatality in SFTS infection are age, high serum aspartate aminotransferase level, prominent coagulation disturbance, presence of neurologic manifestations, and high SFTS viral loads.[4,19] Recently, Li et al.[17] reported that peak viral RNA loads, and IL-6 and IL-10 were significantly lower in non-fatal cases than fatal cases. In addition, Ding et al.[6] described that most fatalities occurred within 2 weeks after onset of illness, in patients older than 70 years of age, and in misdiagnosis with other infectious diseases such as human granulocytic anaplasmosis or hemorrhagic fever with renal syndrome.

\section{DIAGNOSIS}

In process of diagnosis of SFTS, a clinical suspicion based on symptoms such as fever, gastrointestinal manifestation, neurologic disturbance, and hematologic abnormalities is critical along with history and inspection of tick bite. Because tick bite is not evident in all SFTS infection, outside activity and contact with animals should be evaluated. In diagnosis of SFTS, it is also important to perform differential diagnosis with other infectious diseases such as anaplasmosis, hemorrhagic fever with renal syndrome, leptospirosis, and tsutsugamushi diseases. When tick bite history is evident in patients, tsutsugamushi disease is the first disease for differential diagnosis in Korea due to its relatively increasing prevalence rate in a similar seasonal distribution to SFTS.

Laboratory diagnosis of SFTS virus is based on the presence of at least 1 of 3 following criteria that are viral isolation, viral identification by reverse transcriptase polymerase chain reaction (RT-PCR), or serologic detection of 4-fold increase in anti-SFTS virus immunoglobulin $\mathrm{G}$ titers between acute and convalescent phases.[13] Viral RNA can be isolated after inoculation with Vero cells or DH82 cells from patient's serum.[8] Detection limit of viral load using quantitative real time RT-PCR is 10 copies $/ \mu \mathrm{L}$, and PCR method is used to detect L, M, and S segments of viral RNA with $98 \%$ sensitivity and 99\% specificity.[2] Initial detection of $\mathrm{S}$ segment is usually followed by detection of $\mathrm{L}$ and $\mathrm{M}$ segments of SFTS virus for laboratory confirmation.

\section{TREATMENT AND PREVENTION}

Despite various reports on treatment, specific therapeutic guidelines for SFTS have not been established. Supportive management along with intensive monitoring are the mainstream of treatment. Even though ribavirin was recognized as an effective therapy for Crimean-Congo hemorrhagic fever and hemorrhagic fever with renal syndrome, it was recently reported that effectiveness of intravenous ribavirin on disease fatality lacked evidence in treatment of SFTS. $[19,20]$ It was reported that cytokine-mediated inflammatory response involving IL-1 $\beta$, IL-8, macrophage inflammatory protein $1 \beta$, and IFN $-\gamma$ is a potential immunologic mechanism in disease progression and associated with disease severity; consequently, plasma exchange and corticosteroid were reported to be efficient in suppressing cytokine storm.[4,19,21] However, use of corticosteroid is still controversial in treating patients with SFTS because it potentially increases the risk of developing other critical diseases and secondary infections by increasing viral concentration.[19] As effectively used in Crimean-Congo hemorrhagic fever, intravenous immunoglobulin can be used to suppress macrophage activation and cytokine storm.[22] Among various conservative treatment, Oh et al.[21] recently reported that a combination of plasma exchange and oral ribavirin showed effectiveness on severe cases of SFTS. Furthermore, because multiorgan dysfunction and failures are common in clinical course of more severe cases of SFTS, symptomatic management are used in treating complications such as acute lung injury, acute respiratory distress syndrome, disseminated intravascular coagulation, renal failure, and cardiovascular disturbances. A prospective case-control study is highly required to evaluate the effectiveness and duration of each therapeutic modality in treating SFTS.

In terms of prevention of SFTS, vaccines or prophylactic drugs have not been introduced yet. The best measure for prevention is to reduce outside activity and to wash body and clothing thoroughly after outside activity. If ticks are detected on body surface, it is critical not to forcefully detach tick off the body because the remaining parts of ticks, once not fully detached, can continuously serve as sources of viral transmission. Therefore, it is best to find nearby medical facilities when tick bite is suspected. When SFTS infection is confirmed, the patient is recommended for isolation, and healthcare workers are advised to wear personal protective equipment to prevent human-to-human transmission.

\section{CONCLUSION}

This systemic review highlights epidemiological and clinical features of SFTS as well as current information on diagnosis and treatment. However, in-depth studies on pathogenesis, route of transmission, clinical features, and treatment are required be- 
cause other infectious diseases manifest similar symptoms to SFTS. Recently, Heartland virus, a newly identified species of Phlebovirus transmitted by Amblyomma americanum in the United States, is reported to cause fever, leukopenia, and thrombocytopenia, which share initial clinical manifestations with SFTS.[23] Because SFTS endemic areas continue to expand out of China to nearby countries such as Korea and Japan, development of specific antiviral treatment and efficient vaccines needs prompt attention as well as surveillance program.

\section{REFERENCES}

1) Gai ZT, Zhang Y, Liang MF, Jin C, Zhang S, Zhu CB, et al: Clinical progress and risk factors for death in severe fever with thrombocytopenia syndrome patients. J Infect Dis 2012; 206: 1095-102.

2) Liu S, Chai C, Wang C, Amer S, Lv H, He H, et al: Systematic review of severe fever with thrombocytopenia syndrome:virology, epidemiology, and clinical characteristics. Rev Med Virol 2014; 24: 90-102.

3) Takahashi T, Maeda K, Suzuki T, Ishido A, Shigeoka T, Tominaga $\mathrm{T}$, et al: The first identification and retrospective study of severe Fever with thrombocytopenia syndrome in Japan. J Infect Dis 2014; 209: 816-27.

4) Zhang YZ, He YW, Dai YA, Xiong Y, Zheng H, Zhou DJ, et al: Hemorrhagic fever caused by a novel Bunyavirus in China: pathogenesis and correlates of fatal outcome. Clin Infect Dis 2012; 54: 527-33.

5) Yu XJ, Liang MF, Zhang SY, Liu Y, Li JD, Sun YL, et al: Fever with thrombocytopenia associated with a novel bunyavirus in China. N Engl J Med 2011; 364: 1523-32.

6) Ding F, Zhang W, Wang L, Hu W, Soares Magalhaes RJ, Sun $\mathrm{H}$, et al: Epidemiologic features of severe fever with thrombocytopenia syndrome in China, 2011-2012. Clin Infect Dis 2013; 56: 1682-3.

7) Denic S, Janbeih J, Nair S, Conca W, Tariq WU, Al-Salam S: Acute Thrombocytopenia, Leucopenia, and Multiorgan Dysfunction: The First Case of SFTS Bunyavirus outside China? Case Rep Infect Dis 2011; 2011: 204056.

8) Kim KH, Yi J, Kim G, Choi SJ, Jun KI, Kim NH, et al: Severe fever with thrombocytopenia syndrome, South Korea, 2012. Emerg Infect Dis 2013; 19: 1892-4.

9) Chang MS, Woo JH: Severe fever with thrombocytopenia syndrome: tick-mediated viral disease. J Korean Med Sci 2013; 28: 795-6.

10) Korea Center for Disease Control and Prevention: A Current
2014 Mar [2014 Mar 18]. Available from:http://cdc.go.kr/CDC/intro/CdcKrIntro0201.jsp?menuIds=HOME001-MNU1154-MN U0005-MNU0011\&cid=21944.

11) Zhang YZ, Zhou DJ, Qin XC, Tian JH, Xiong Y, Wang JB, et al: The ecology, genetic diversity, and phylogeny of Huaiyangshan virus in China. J Virol 2012; 86: 2864-8.

12) Zhao L, Zhai S, Wen H, Cui F, Chi Y, Wang L, et al: Severe fever with thrombocytopenia syndrome virus, Shandong Province, China. Emerg Infect Dis 2012; 18: 963-5.

13) Tang $\mathrm{X}, \mathrm{Wu} \mathrm{W}$, Wang $\mathrm{H}$, Du Y, Liu L, Kang K, et al: Human-to-human transmission of severe fever with thrombocytopenia syndrome bunyavirus through contact with infectious blood. J Infect Dis 2013; 207: 736-9.

14) Bao CJ, Guo XL, Qi X, Hu JL, Zhou MH, Varma JK, et al: A family cluster of infections by a newly recognized bunyavirus in eastern China, 2007: further evidence of person-to-person transmission. Clin Infect Dis 2011; 53: 1208-14.

15) Gai Z, Liang M, Zhang Y, Zhang S, Jin C, Wang SW, et al: Person-to-person transmission of severe fever with thrombocytopenia syndrome bunyavirus through blood contact. Clin Infect Dis 2012; 54: 249-52.

16) Heo ST, Cheon MS, Kim JW: Four cases of severe fever with thrombocytopenia syndrome occurring in Jeju. Korean $\mathrm{J}$ Dermatol 2014; 52: 173-7.

17) Li J, Han Y, Xing Y, Li S, Kong L, Zhang Y, et al: Concurrent measurement of dynamic changes in viral load, serum enzymes, T cell subsets, and cytokines in patients with severe Fever with thrombocytopenia syndrome. PLoS One 2014; 9: e91679.

18) Deng B, Zhang S, Geng Y, Zhang Y, Wang Y, Yao W, et al: Cytokine and chemokine levels in patients with severe fever with thrombocytopenia syndrome virus. PLoS One 2012; 7: e41365.

19) Deng B, Zhou B, Zhang S, Zhu Y, Han L, Geng Y, et al: Clinical features and factors associated with severity and fatality among patients with severe fever with thrombocytopenia syndrome Bunyavirus infection in Northeast China. PLoS One 2013; 8: e80802.

20) Liu W, Lu QB, Cui N, Li H, Wang LY, Liu K, et al: Case-fatality ratio and effectiveness of ribavirin therapy among hospitalized patients in china who had severe fever with thrombocytopenia syndrome. Clin Infect Dis 2013; 57: 1292-9.

21) Oh WS, Heo ST, Kim SH, Choi WJ, Han MG, Kim JY: Plasma exchange and ribavirin for rapidly progressive severe fever with thrombocytopenia syndrome. Int J Infect Dis 2014; 18: 84-6. 
22) Erduran E, Bahadir A, Palanci N, Gedik Y: The treatment of crimean-congo hemorrhagic fever with high-dose methylprednisolone, intravenous immunoglobulin, and fresh frozen plasma. J Pediatr Hematol Oncol 2013; 35: e19-24.
23) Pastula DM, Turabelidze G, Yates KF, Jones TF, Lambert AJ, Panella AJ, et al: Notes from the field: heartland virus disease - United States, 2012-2013. MMWR Morb Mortal Wkly Rep 2014; 63: 270-1. 